\title{
Confirmation of TNIP1 and IL23A as susceptibility loci for psoriatic arthritis
}

\author{
John Bowes, ${ }^{1}$ Gisela Orozco, ${ }^{1}$ Edward Flynn, ${ }^{1}$ Pauline Ho ${ }^{1,2}$ Rasha Brier, ${ }^{1}$ \\ Helena Marzo-Ortega, ${ }^{3}$ Laura Coates, ${ }^{3}$ Ross McManus, ${ }^{4}$ Anthony W Ryan, ${ }^{4}$ \\ David Kane, ${ }^{5}$ Eleanor Korendowych, ${ }^{6}$ Neil McHugh, ${ }^{6}$ Oliver FitzGerald, ${ }^{7}$ \\ Jonathan Packham, ${ }^{8,9}$ Ann W Morgan, ${ }^{3}$ Ian N Bruce, ${ }^{1,2}$ Anne Barton ${ }^{1,2}$
}

\section{- Additional data}

(supplementary tables) are published online only. To view these files please visit the journal online at http://ard.bmj.com.

${ }^{1}$ Arthritis Research UK Epidemiology Unit, Manchester Academic Health Science Centre, The University of Manchester, Manchester, UK

${ }^{2}$ The Kellgren Centre for

Rheumatology, Central

Manchester Foundation

Trust, NIHR Manchester

Biomedical Research Centre,

Manchester, UK

${ }^{3}$ NIHR-Leeds Musculoskeletal

Biomedical Research Unit, Leeds

Institute of Molecular Medicine,

University of Leeds, Leeds, UK

${ }^{4}$ Department of Clinical

Medicine, Institute of Molecular

Medicine, Trinity College Dublin,

Dublin, Ireland

${ }^{5}$ Adelaide and Meath Hospital and Trinity College Dublin,

Dublin, Ireland

${ }^{6}$ Royal National Hospital for

Rheumatic Diseases and

Department of Pharmacy and

Pharmacology, University of

Bath, Bath, UK

${ }^{7}$ Department of Rheumatology, St Vincent's University Hospital, UCD School of Medicine and

Medical Sciences and Conway Institute of Biomolecular and

Biomedical Research, University

College Dublin, Dublin, Ireland

${ }^{8}$ Haywood Hospital, Stoke on

Trent, UK

${ }^{9}$ Arthritis Research UK Primary Care Centre, Keele University, Keele, UK

\section{Correspondence to \\ Professor Anne Barton, Arthritis Research UK, \\ Stopford Building, University of \\ Manchester, Manchester, UK; \\ Accepted 24 April 2011 \\ Published Online First \\ 29 May 2011}

anne.barton@manchester.ac.uk

This paper is freely available online under the BMJ Journals unlocked scheme, see http:// ard.bmj.com/info/unlocked.dtl

\section{ABSTRACT}

Objectives To investigate a shared genetic aetiology for skin involvement in psoriasis and psoriatic arthritis (PsA) by genotyping single-nucleotide polymorphisms (SNPs), reported to be associated in genome-wide association studies of psoriasis, in patients with PSA.

Methods SNPs with reported evidence for association with psoriasis were genotyped in a PsA case and control collection from the UK and Ireland. Genotype and allele frequencies were compared between PsA cases and controls using the Armitage test for trend.

Results Seven SNPS mapping to the IL1RN, TNIP1, TNFAIP3, TSC1, IL23A, SMARCA4 and RNF114 genes were successfully genotyped. The IL23A and TNIP1 genes showed convincing evidence for association (rs2066808, $p=9.1 \times 10^{-7} ;$ rs 17728338, $p=3.5 \times$ $10^{-5}$, respectively) whilst SNPs mapping to the TNFAIP3, TSC1 and RNF114 genes showed nominal evidence for association (rs610604, $p=0.03$; rs1076160, $p=0.03$; rs495337, $p=0.0025)$. No evidence for association with IL1RN or SMARCA4 was found but the power to detect association was low.

Conclusions SNPS mapping to previously reported psoriasis loci show evidence for association to PSA, thus supporting the hypothesis that the genetic aetiology of skin involvement is the same in both PsA and psoriasis.

Psoriatic arthritis (PsA) and psoriasis are both complex diseases with genetic and environmental contributions to their aetiology. Estimates suggest that the genetic contribution to PsA may be higher than psoriasis yet most work has been done on the latter given its greater population prevalence. ${ }^{1}$

Both diseases are characterised by psoriasis skin involvement; if the psoriasis is aetiologically identical, then all confirmed psoriasis susceptibility loci should also be associated with PsA. Of the psoriasis susceptibility loci studied to date, the $H L A C w^{*} 0602$ allele and variants mapping to the $I L 23 R$ and IL12B genes have been associated with PsA. ${ }^{2-4}$ It might be postulated that additional PsA susceptibility loci exist. This is supported by evidence that the IL13 gene locus is associated with PsA, but not psoriasis alone. ${ }^{6}{ }^{6}$ Furthermore, the recently identified TRAF3IP2 gene locus shows stronger effect sizes in some, ${ }^{7} 8$ but not all, ${ }^{9}$ PsA populations compared with psoriasis, suggesting that it too may represent a PsA-specific locus. If the skin manifestations of both diseases represent a common phenotypic endpoint arising from different aetiologies, some differences in susceptibility loci would be expected. The identification of risk loci specific to psoriasis would provide compelling evidence that the genetic basis of the skin component of the two diseases was different. There has been some evidence reported to support this possibility. Variants within the LCE gene locus were reported to be associated with psoriasis but not PsA in a German cohort, ${ }^{10}$ but a subsequent study in a population from the British Isles showed association with PsA suggesting it is involved in psoriasis per se. ${ }^{11}$

Genome-wide association studies (GWAS) have resulted in the identification of further psoriasis susceptibility loci. ${ }^{12}{ }^{13}$ This provides an opportunity to explore further the overlap in genetic susceptibility between psoriasis and PsA. If the genetic liability towards skin involvement is shared between the two diseases, then loci identified in psoriasis GWAS would also show evidence of association of the susceptibility with PsA.

\section{METHODS \\ Patient samples}

One thousand and fifty-seven genomic DNA samples were collected from Caucasian PsA patients recruited from the UK and Ireland (885 UK and 172 Ireland), the details of which have previously been described ${ }^{14-16}$ and phenotype details are summarised in supplementary table 4 (available online only). PsA was defined as the presence of both psoriasis and peripheral inflammatory arthritis irrespective of rheumatoid factor status. All subjects provided informed consent and the study was approved by the North West Multicentre Research Ethics Committee (MREC 99/8/84).

\section{Control data}

The data for healthy UK controls was sourced from the Wellcome Trust Case-Control Consortium 2 (WTCCC2) project (http://www.wtccc.org.uk). This project genotyped over 5000 individuals from the 1958 British Birth Cohort and the UK Blood Service Collection for the Illumina Human1M-Duo and the Affymetrix Human SNP Array 6.0. If control data for any single-nucleotide polymorphism (SNP) were not available from the WTCCC2 then it was generated in-house using DNA from 2104 1958 British Birth Cohort samples. In addition, 375 control samples were available from Ireland for genotyping. 


\section{SNP selection}

Seven SNPs were selected from two published psoriasis GWAS available at the time of study design. ${ }^{12}{ }^{13}$ Four of the selected SNPs: rs17728338 (TNIP1); rs2066808 (IL23A); rs610604 (TNFAIP3) and rs495337 (RNF114) all reached genome-wide significance in the reported studies. Three further SNPs: rs 1076160 (TSC1); rs12983316 (SMARCA4) and rs397211 (IL1RN) reached modest levels of significance.

\section{Genotyping}

Genotyping of the SNPs was performed using Sequenom's MassARRAY system (San Diego, California, USA) according to the manufacturers' specifications using 10 ng of genomic DNA for all PsA patients, the Ireland control samples and 1958 British Birth Cohort samples (where necessary).

\section{Statistical analysis}

All quality control steps and statistical analyses were performed using the PLINK package. ${ }^{17}$ SNPs and samples with greater than $10 \%$ missing data were excluded from the study. Test statistics were calculated for deviation from Hardy-Weinberg equilibrium using an exact test, the Cochran-Armitage trend test and OR (including $95 \% \mathrm{CI}$ ). A p value of less than $10^{-4}$ was considered statistically significant following recommendations that this should be the threshold used for claims of confirmed association when the same SNP has been shown to be associated at genomewide significance levels in another disease. ${ }^{18}$ Power calculations were performed using the online genetic power calculator. ${ }^{19}$

The primary analysis, from which conclusions regarding support for validation will be drawn, consisted of the joint analysis of both the UK and Ireland as a single population. As the differing population history could introduce bias due to population structure, we attempted to control this explicitly by analysing the UK and Ireland datasets separately followed by inversevariance meta-analysis under the assumption of fixed effects. Allelic heterogeneity between the two groups was estimated using the Cochran $\mathrm{Q}$ and $\mathrm{I}^{2}$ statistics.

Exploratory analysis of subphenotypes were performed on the UK dataset based on the age at onset of psoriasis (type I psoriasis has an onset $\leq 40$ years of age while type II psoriasis is defined as an onset $>40$ years of age) and seronegativity for rheumatoid factor (to exclude those patients who may have psoriasis and co-existent rheumatoid arthritis).

\section{RESULTS}

\section{Genotyping}

WTCCC2 control data were available for all SNPs except rs495337; this SNP was therefore genotyped in-house. All genotyped SNPs demonstrated good, well resolved clusters.

\section{Statistical analysis}

Genotype frequencies for six SNPs conformed to HardyWeinberg expectations, but rs610604 (TNFAIP3) showed moderate deviation. SNPs mapping to the IL23A and TNIP1 genes showed confirmed evidence for association with PsA at the stringent significance values set (rs2066808, IL23A, OR 0.54 (0.43-0.70), $\mathrm{p}=9.1 \times 10^{-7}$; rs17728338, TNIP1, OR 1.49 $(1.23-1.80) ; p=3.5 \times 10^{-5}$ ) (table 1). No qualitative differences were noted when the UK and Ireland datasets were analysed separately for these two SNPs (supplementary tables 1 and 2, available online only). Meta-analysis of the two datasets support the results from the primary analysis, with only rs610604 (TNFAIP3) demonstrating significant heterogeneity between the 


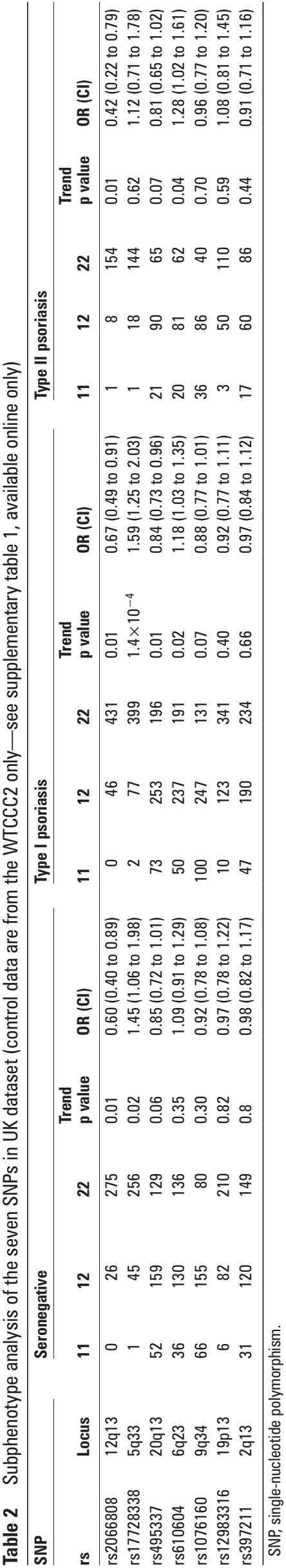

two populations (supplementary table 3, available online only). The association of the TNIP1 SNP, rs17728338, appeared stronger in samples from PsA patients with type I psoriasis $(n=478)$ with a much lower OR observed in the PsA samples with type II psoriasis ( $\mathrm{n}=163$ ) (OR 1.59 vs 1.12, respectively), although the CI overlap (table 2). However, given the limited sample numbers these results should be considered with caution. There were no qualitative differences in the effects of any SNPs when the analysis was limited to seronegative individuals $(\mathrm{n}=302)$.

SNPs mapping to the TNFAIP3, TSC1 and RNF114 genes showed borderline evidence for association at a nominal pvalue threshold of 0.05 and the remaining two SNPs showed no evidence for association with PsA in this dataset (table 1).

\section{DISCUSSION}

We have confirmed that two loci, IL23A and TNIP1, associated with psoriasis in multiple populations, are also associated with PsA. Support for another three loci, TNFAIP3, TSC1 and $R N F 114$, is provided by evidence of association at nominal $p$ value thresholds of 0.05 although confirmation of association with PsA will be required in other, large datasets.

SNPs mapping to the final two loci tested, SMARCA4 and IL1RN, did not show any evidence of association with PsA, which might indicate that they are exclusively associated with psoriasis. However, the power to detect association with these loci was poor in the sample size tested $(20 \%$ and $17 \%$, respectively). Interestingly, the risk allele in both the psoriasis GWAS and the current data is the same and the strength and direction of association are similar. Association of TSC1, SMARCA4 and $I L 1 R N$ with psoriasis was reported by Nair et al, ${ }^{12}$ although association was found in two datasets $(p<0.05)$, the strength of association failed to reach genome-wide significance levels in the combined dataset of nearly 13000 samples. No other studies have yet replicated the association of SMARCA4, casting doubt on whether it truly represents a psoriasis susceptibility locus. However, an independent family-based study in a French population has shown association of the IL1RN gene with psoriasis, albeit with a different SNP (rs315934; $\mathrm{r}^{2}$ with rs397211=0.08) ${ }^{20} \mathrm{It}$ may be, therefore, that confirmed evidence for association with PsA will be demonstrated in a larger dataset or by meta-analysis in the future.

Strengths of this study include the large sample size tested, the majority of whom were from the UK. Previous studies have shown minimal population stratification across the UK thus minimising this potential source of bias in genotyping. Differences in genetic substructure between the UK and Irish population have been noted previously, but we controlled for this by analysing the data from each of the populations separately followed by meta-analysis. ${ }^{8}$

Some limitations of the study are apparent, particularly the lack of power to exclude association with PsA confidently for psoriasis loci with small effect sizes. In addition, we have tested a single SNP at each locus based on previous reports of confirmed association with psoriasis. However, this is unlikely to be the causal variant and association at any given locus may be complex. For example, several SNPs mapping to the IL23R and $I L 12 B$ genes show independent associations with psoriasis susceptibility; fine mapping of the associated regions will be required to identify all associated variants and to identify those most likely to be functionally important. A further limitation is the fact that the psoriasis GWAS contain a large proportion of patients with PsA, which makes comparison of the genetic aetiology of the two diseases very difficult. Studies comparing the genetic aetiology of the two diseases would be 
greatly facilitated by a psoriasis cohort reviewed by a rheumatologist to exclude patients with inflammatory arthritis. It should also be noted that we have used a broad definition of PsA, including patients with psoriasis and a peripheral inflammatory arthritis, rather than the recently validated CASPAR criteria. ${ }^{21}$ The latter include $\mathrm{x}$-ray changes as part of the criteria and this was not available for many subjects as cohort collections were started before the introduction of these criteria. However, the majority of patients did satisfy these criteria, when x-ray data were available. Finally, a number of GWAS of psoriasis have been published recently in European and Asian populations providing a further opportunity to explore whether psoriasis susceptibility loci are also associated with PsA; 89122223 however, this information was not available at the time of study design and so the novel psoriasis loci identified from those scans still require investigation for association with PsA.

It is expected that epistasis between genetic loci may result in larger effect sizes. Indeed, the first demonstration of this was recently reported in that association of ERAP1 with psoriasis was only observed in individuals carrying the HLA Cw*0602 allele. ${ }^{8}$ No interaction was detected between either of the TNIP1 or IL23A markers and HLA CW*0602 (data not shown). However, interaction analyses would be best explored in larger sample collections that would afford greater power than that provided in the current study.

In summary, our data largely support the thesis that psoriasis susceptibility loci are also associated with PsA; in particular, we show definite confirmation that IL23A and TNIP1 and supportive evidence that the TNFAIP3, TSC1 and RNF114 genes are associated with susceptibility to PsA as well as psoriasis.

Acknowledgements This study makes use of data generated by the Wellcome Trust Case-Control Consortium. The authors are grateful for access to DNA from the 1958 Birth Cohort

Funding The authors acknowledge the support of the NIHR Manchester Biomedical Research Centre and NIHR Leeds Musculoskeletal Biomedical Research Unit. JB, INB and $A B$ are funded by Arthritis Research UK (grant 17552). EF is supported by the European Community's Sixth Framework Programme AutoCure funding. This work was funded by the Arthritis Research UK. Funding for the project was provided by the Wellcome Trust under awards 076113 and 085475.

Patient consent Obtained.

Ethics approval This study was conducted with the approval of the North West Multicentre Research Ethics Committee (MREC 99/8/84).

Provenance and peer review Not commissioned; externally peer reviewed.

\section{REFERENCES}

1. Karason A, Love TJ, Gudbjornsson B. A strong heritability of psoriatic arthritis over four generations - the Reykjavik Psoriatic Arthritis Study. Rheumatology (Oxford) 2009;48:1424-8
2. Ho PY, Barton A, Worthington J, et al. Investigating the role of the HLA$\mathrm{CW}^{*} 06$ and HLA-DRB1 genes in susceptibility to psoriatic arthritis: comparison with psoriasis and undifferentiated inflammatory arthritis. Ann Rheum Dis 2008;67:677-82

3. Filer C, Ho P, Smith RL, et al. Investigation of association of the IL12B and IL23R genes with psoriatic arthritis. Arthritis Rheum 2008;58:3705-9.

4. Hüffmeier U, Lascorz J, Böhm B, et al. Genetic variants of the IL-23R pathway: association with psoriatic arthritis and psoriasis vulgaris, but no specific risk factor for arthritis. J Invest Dermatol 2009;129:355-8.

5. Duffin KC, Freeny IC, Schrodi SJ, et al. Association between IL13 polymorphisms and psoriatic arthritis is modified by smoking. J Invest Dermatol 2009:129:2777-83.

6. Bowes J, Eyre S, Flynn E, et al. Evidence to support IL-13 as a risk locus for psoriatic arthritis but not psoriasis vulgaris. Ann Rheum Dis 2011;70:1016-19.

7. Hüffmeier $\mathbf{U}$, Uebe $S$, Ekici $A B$, et al. Common variants at TRAF3IP2 are associated with susceptibility to psoriatic arthritis and psoriasis. Nat Genet 2010;42:996-9.

8. Strange A, Capon F, Spencer CC, et al. A genome-wide association study identifies new psoriasis susceptibility loci and an interaction between HLA-C and ERAP1. Nat Genet 2010;42:985-90.

9. Ellinghaus $\mathbf{E}$, Ellinghaus D, Stuart PE, et al. Genome-wide association study identifies a psoriasis susceptibility locus at TRAF3IP2. Nat Genet 2010;42:991-5.

10. Hüffmeier $\mathbf{U}$, Estivill $X$, Riveira-Munoz $E$, et al. Deletion of $\angle C E 3 C$ and $\angle C E 3 B$ genes at PSORS4 does not contribute to susceptibility to psoriatic arthritis in German patients. Ann Rheum Dis 2010;69:876-8.

11. Bowes J, Flynn $\mathrm{E}, \mathrm{H}_{0} \mathrm{P}$, et al. Variants in linkage disequilibrium with the late cornified envelope gene cluster deletion are associated with susceptibility to psoriatic arthritis. Ann Rheum Dis 2010;69:2199-203.

12. Nair RP, Duffin KC, Helms C, et al. Genome-wide scan reveals association of psoriasis with IL-23 and NF-kappaB pathways. Nat Genet 2009;41:199-204.

13. Capon F, Bijlmakers MJ, Wolf N, et al. Identification of ZNF313/RNF114 as a nove psoriasis susceptibility gene. Hum Mol Genet 2008;17:1938-45.

14. Ho P, Bruce IN, Silman A, et al. Evidence for common genetic control in pathways of inflammation for Crohn's disease and psoriatic arthritis. Arthritis Rheum 2005:52:3596-602.

15. Al-Heresh AM, Proctor J, Jones SM, et al. Tumour necrosis factor-alpha polymorphism and the $\mathrm{HLA}-\mathrm{CW}^{*} 0602$ allele in psoriatic arthritis. Rheumatology (Oxford) 2002;41:525-30.

16. Balding J, Kane D, Livingstone W, et al. Cytokine gene polymorphisms: association with psoriatic arthritis susceptibility and severity. Arthritis Rheum 2003:48:1408-13.

17. Purcell S, Neale B, Todd-Brown K, et al. PLINK: a tool set for wholegenome association and population-based linkage analyses. Am J Hum Genet 2007;81:559-75.

18. Smyth DJ, Plagnol V, Walker NM, et al. Shared and distinct genetic variants in type 1 diabetes and celiac disease. N Engl J Med 2008;359:2767-77.

19. Purcell S, Cherny SS, Sham PC. Genetic power calculator: design of linkage and association genetic mapping studies of complex traits. Bioinformatics 2003;19:149-50.

20. Oudot T, Lesueur F, Guedj M, et al. An association study of 22 candidate genes in psoriasis families reveals shared genetic factors with other autoimmune and skin disorders. J Invest Dermato/ 2009;129:2637-45

21. Taylor W, Gladman D, Helliwell $P$, et al. Classification criteria for psoriatic arthritis: development of new criteria from a large international study. Arthritis Rheum 2006;54:2665-73.

22. Sun LD, Cheng $H$, Wang ZX, et al. Association analyses identify six new psoriasis susceptibility loci in the Chinese population. Nat Genet 2010:42:1005-9.

23. Stuart PE, Nair RP, Ellinghaus E, et al. Genome-wide association analysis identifies three psoriasis susceptibility loci. Nat Genet 2010;42:1000-4. 